\title{
New results on quasinormal modes of anti-de Sitter black holes
}

\section{Jaqueline Morgan*}

Universidade Federal do $A B C$

E-mail: jaqueline.morgandufabc.edu.br

\author{
Alex S. Miranda \\ Instituto de Física \\ Universidade Federal do Rio de Janeiro \\ E-mail: astmirandadif.ufrj.br
}

Vilson T. Zanchin

Universidade Federal do ABC

E-mail: zanchin@ufabc.edu.br

In this work we study the quasinormal spectra of gravitational perturbations of $d$-dimensional plane-symmetric AdS black holes in the context of the AdS/CFT correspondence. The perturbation equations are written for two different gauge-invariant variables: in the first the variables are chosen in such a way to put the radial part of the fundamental equations into a Schrödinger-like form; in the second we have a new set of fundamental variables where the imposition of Dirichlet boundary conditions at infinity leads exactly to the quasinormal frequencies associated to black hole here studied. The AdS/CFT correspondence helps one to find an appropriate condition to be applied to each set of variables at the AdS space boundary in order to produce identical QNM spectra for a given sector of perturbations, and more, that the quasinormal modes will correspond to the poles of the stress-energy tensor correlators in the dual field theory. We investigate analytically the quasinormal spectrum for small wavenumbers, large frequencies, and large number of spacetime dimensions. Using series solutions, we obtain numerically the dispersion relations of the first few modes in the low-, intermediate- and high-wavenumber regimes, and we analyzed the eikonal limit.

5th International School on Field Theory and Gravitation, April 20 - 242009

Cuiabá city, Brazil

\footnotetext{
* Poster Session
} 


\section{Introduction}

Theoretical studies on black holes in asymptotically anti-de Sitter spacetimes have attracted substantial attention since the advent of the anti-de Sitter/conformal field theory (AdS/CFT) correspondence [1]. Such a correspondence has established a mapping between quantities in the bulk AdS black-hole physics and observables in a boundary finite-temperature field theory, and appears to be valid for an arbitrary number of spacetime dimensions, extrapolating their original formulations. According to the AdS/CFT correspondence, an asymptotically AdS black hole is, in the CFT side, associated to a system in thermal equilibrium whose temperature is the Hawking temperature of the black hole. In such a context, black hole perturbations correspond to small deviations from equilibrium of the CFT thermal system, and the characteristic damping time of perturbations, which is given by the inverse of the imaginary part of the fundamental QNM frequency, is a measure of the dynamical timescale of approach to thermal equilibrium of the corresponding conformal field theory [阴].

There are important issues in the study of the vibrational modes of AdS black branes to be analyzed in a fully $d$-dimensional context. We can mention the arbitrariness in the choice of gauge-invariant combinations of metric variations as fundamental variables of the gravitational perturbations, and the ambiguity in defining an appropriate condition for the quasinormal modes at

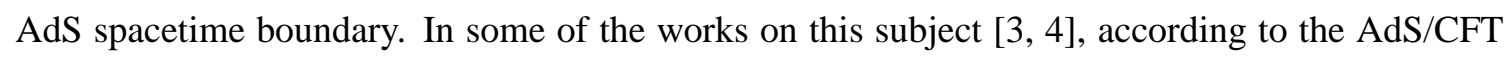
duality, the ambiguities characteristic of classical-field dynamics at AdS spacetimes were eliminated by defining the quasinormal $(\mathrm{QN})$ frequencies as the poles, in the space of frequency and momentum, of retarded Green functions in the dual field theory. These and other related subjects are investigated here considering AdS black holes in spacetimes of arbitrary number of dimensions.

\section{The background spacetime}

The background spacetime considered here represents a $d$-dimensional plane-symmetric asymptotically anti-de Sitter (AdS) black hole [5, 5]. The spacetime can be locally written as a product of a two-dimensional spacetime $\mathscr{N}^{2}$, spanned by a timelike coordinate $t$ and a radial spacelike coordinate $u=r_{h} / r$, and a $(d-2)$-dimensional space $\mathscr{K}^{d-2}$ with constant sectional curvature $K=0$ [7, 8]. With such a decomposition, the background metric in Schwarzschild-like coordinates takes the form

$$
d s^{2}=\frac{r_{h}^{2}}{u^{2} R^{2}}\left[-f(u) d t^{2}+\sum_{i=2}^{d-1} d x^{i} d x_{i}\right]+\frac{R^{2}}{u^{2} f(u)} d u^{2},
$$

for which $f(u)=1-u^{d-1}$ with $r_{h}$ being the event horizon radius. The Hawking temperature of the black brane is $T=(d-1) r_{h} / 4 \pi R^{2}$, where $R$ is the AdS radius. The coordinates $x^{i}, i=2,3, \ldots, d-1$, span the $\mathscr{K}^{d-2}$ space.

The radial coordinate $u$ covers, without singularities, the whole region of interest for the analysis of the QNM of the AdS black hole of metric (2.1), namely, the range $u \in(1,0)$. The event horizon is located at $u=1$, and the AdS spatial infinity $(r \rightarrow \infty)$ is at $u=0$. 


\section{Fundamental equations for the gravitational perturbations}

Following the procedure presented in Ref. [7], the gravitational perturbations are grouped into three distinct classes (sectors) that can be tensorial, vectorial, or scalar perturbations, corresponding respectively to the scalar, shear and sound symmetry channels for the gravitational fluctuations considered in Ref. [3]. Here we present the fundamental equations for two kinds of variables in $d$ spacetime dimensions, the Kovtun-Starinets (KS) and Regge-Wheeler-Zerilli (RWZ) variables.

\subsection{Master equations for the RWZ variables}

Kodama and Ishibashi [8] showed that for a black brane in four or more spacetime dimensions, the Einstein equations for the gravitational perturbations can be reduced to three independent second-order wave equations in a two-dimensional static spacetime, one equation corresponding to each one of the perturbation modes. After Fourier decomposition of such perturbation functions, $\Phi_{p}(t, u)=\int \Phi_{p}(u) e^{i \omega t} d \omega$, the perturbation equations take a Schrödinger-like form,

$$
\frac{d^{2} \Phi_{p}}{d \mathfrak{r}_{*}^{2}}+\left(\mathfrak{w}^{2}-V_{p}\right) \Phi_{p}=0
$$

where $\mathfrak{r}_{*}$ is the normalized tortoise radial coordinate, defined by $d u / d \mathfrak{r}_{*}=-f(u)$. The label $p$ can be $T, V$ or $S$ depending of the perturbation sector: tensorial, vectorial and scalar, respectively. $V_{p}$ is the effective potential, and the parameter $\mathfrak{w}$ is the normalized frequency defined by $\mathfrak{w}=$ $[(d-1) \omega] / 4 \pi T=\left(R^{2} \omega\right) / r_{h}$, where $T$ stands for the Hawking temperature of the black brane. For example the tensorial effective potential is given by

$$
V_{T}(u)=f(u)\left[\mathfrak{q}^{2}+\frac{d(d-2)}{4 u^{2}}+\frac{(d-2)^{2} u^{d-3}}{4}\right]
$$

where the parameter $\mathfrak{q}$ is the normalized wavenumber defined by $\mathfrak{q}=[(d-1) k] / 4 \pi T=\left(R^{2} k\right) / r_{h}$. The explicit form of $V_{V}$ and $V_{S}$ can be found in Ref. [9].

\subsection{Master equations for the KS variables}

Another choice of fundamental variables for the gravitational perturbations was suggest by Kovtun and Starinets [⿰]月 . Here the fundamental equation for the tensorial sector for the KS variables in $d$ spacetime dimensions is given by

$$
Z_{T}^{\prime \prime}-\left[\frac{d-1-f}{u f}\right] Z_{T}^{\prime}+\left[\frac{\mathfrak{w}^{2}-\mathfrak{q}^{2} f}{f^{2}}\right] Z_{T}=0
$$

where the primes indicate derivatives with respect to the coordinate $u$, and $f=f(u)$ is the horizon function. The fundamental equations for the vectorial and scalar sectors can be see in [9].

An important characteristic of classical field evolutions on asymptotically AdS spacetimes is the variety of choices for the boundary conditions at spatial infinity. In general, these can be Dirichlet, Neumann or Robin boundary conditions. So we need to establish an objective criterion before to choose a specific condition. In the AdS/CFT context, a natural criterion is such that the QNM frequencies of a certain field correspond to poles of two-point correlation functions of the dual 


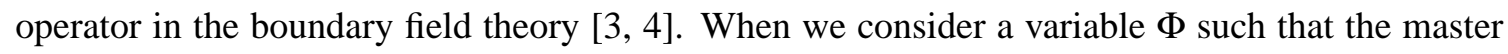
equation for the gravitational perturbations is of the form (3.1), in general the Dirichlet condition at spatial infinity is the 'correct' boundary condition. There is only one exception: for scalar-type perturbations in four spacetime dimensions, the boundary condition that leads to QNM frequencies corresponding to poles of retarded correlation functions is of Robin type. However, when we consider the variable $Z_{p}$ the Dirichlet condition at spatial infinity leads to QNM frequencies corresponding to poles of retarded correlation functions for all cases [9, 10].

\section{The spectra quasinormal}

\subsection{Small wavenumbers, large frequencies}

There is an alternative analysis for large frequencies with finite wavenumbers, namely $\mathfrak{w} \gg \mathfrak{q}$. To first order approximation such a condition is equivalent to the asymptotic limit $\mathfrak{q} \rightarrow 0$, as far as all the other parameters of the model are kept fixed. For all of the perturbation equations with KS variables we have

$$
Z_{p}^{\prime \prime}-\left[\frac{d-1-f}{u f}\right] Z_{p}^{\prime}+\frac{\mathfrak{w}^{2}}{f^{2}} Z_{p}=0
$$

where $p$ denotes the perturbative sector, as already indicated. It is obvious that Eq. (4.1) necessarily imply in identical non-hydrodynamic quasinormal frequencies at $\mathfrak{q}=0$ for all of the perturbation types. With this result we conclude that the dispersion relations for large frequencies are the same for all the three perturbation sectors of a black brane. It is worth noticing here the very good agreement between the analytical and the numerical results as we can see in Table 1 .

\begin{tabular}{ccccccc}
\hline \hline & \multicolumn{2}{c}{ Tensorial } & \multicolumn{2}{c}{ Vectorial } & \multicolumn{2}{c}{ Scalar } \\
\hline$d$ & $\mathfrak{w}_{R}$ & $\mathfrak{w}_{I}$ & $\mathfrak{w}_{R}$ & $\mathfrak{w}_{I}$ & $\mathfrak{w}_{R}$ & $\mathfrak{w}_{I}$ \\
\hline 4 & - & - & 1.84942 & 2.66385 & 1.84942 & 2.66385 \\
5 & 3.11945 & 2.74668 & 3.11945 & 2.74667 & 3.11945 & 2.74668 \\
6 & 4.13591 & 2.69339 & 4.13591 & 2.69339 & 4.13591 & 2.69339 \\
7 & 5.00747 & 2.61247 & 5.00760 & 2.61266 & 5.00758 & 2.61249 \\
\hline \hline
\end{tabular}

Table 1: The frequencies of the first non-hydrodynamic QNM for all perturbation types, calculated with $\mathfrak{q}=0$.

\subsubsection{Large number of spacetime dimensions}

In this section we analyze the perturbation equations when the number of spacetime dimensions is large, namely $d \rightarrow \infty$ with finite $\mathfrak{w}$ and $\mathfrak{q}$. For simplicity, in this analysis we consider the master equations for the RWZ gauge-invariant variables (Eq. (3.1)), in which case the analysis reduces to investigate the asymptotic form of the effective potentials in the limit $d \gg 4$. We thus find

$$
V_{T} \rightarrow \frac{d^{2}}{4 u^{2}} f\left(1+u^{d-1}\right), \quad V_{V} \rightarrow \frac{d^{2}}{4 u^{2}} f\left(1-3 u^{d-1}\right), \quad V_{S} \rightarrow \frac{d^{2}}{4 u^{2}} f\left(1+u^{d-1}\right) .
$$

It is seen that in such a limit the tensorial and scalar potentials are the same. Moreover, in the intervening region between the AdS boundary and the horizon $(0<u<1)$, the second term of the 
above expressions within the parentheses tend to zero in the limit $d \rightarrow \infty$, so that the potentials are identical in this region. Moreover, the tensorial, scalar and vectorial potentials approach the same values at the boundaries. These results suggest that the QNM spectra of the three perturbation sectors for large $d$ are identical. This is an important result because it shows the isospectrality of the gravitational QNM of higher-dimensional AdS black holes.

\subsection{Eikonal limit}

In asymptotically AdS spacetimes the eikonal limit is especially interesting, since large- $q$ modes can be very long-lived [11, 12]. A WKB analysis suggests that for the tensor-type gravitational perturbations (and therefore also scalar fields) and $r_{+} / R \gg 1$, the following asymptotic behavior holds

$$
\begin{aligned}
R \omega^{\mathrm{FL}} & =q+\Pi_{n}\left(\frac{r_{+}}{R}\right)^{\frac{2 d-2}{d+1}} q^{-\frac{d-3}{d+1}}, \\
\Pi_{n} & \equiv \frac{1}{2}\left(\sqrt{\pi}(d-1)\left[\frac{d+1}{2}+2 n\right] \frac{\Gamma\left(\frac{3 d-1}{2 d-2}\right)}{\Gamma\left(\frac{1}{d-1}\right)}\right)^{\frac{2 d-2}{d+1}} e^{-\frac{2 i \pi}{d+1}},
\end{aligned}
$$

as $q \rightarrow \infty$ [10, 12]. So large- $q$ modes are very long-lived, and they could play a prominent role in the BH's response to generic perturbations.

Our numerical results are consistent with the $q^{-(d-3) /(d+1)}$ dependence of the characteristic frequencies, for $d=4,5$, and 6 . Our results are also highly consistent with the functional dependence on $r_{+}, q$ as given by equation (4.3). Then, we assume the power-law behavior (4.3) in $q$ and $r_{+}$, and fit the numerical results to the following function

$$
R \omega^{\mathrm{Num}}=q+\left(a_{R} \operatorname{Re}\left[\Pi_{n}\right]+i a_{I} \operatorname{Im}\left[\Pi_{n}\right]\right)\left(\frac{r_{+}}{R}\right)^{\frac{2 d-2}{d+1}} q^{-\frac{d-3}{d+1}},
$$

thereby testing the prefactor in (4.3). Our results in this analysis are strong indicators that Eq.(4.3) is consistent with all our numerical results for scalar fields or tensor-type gravitational, but does not account for the correct quantitative behavior of these weakly-damped modes for the vectorial and scalar sectors. Though the equation (4.3) captures the essential qualitative behavior with $r_{+}$, $q$ for vector-type (see Fig. 1) and scalar-type gravitational perturbations it only can describe quantitatively the numerical results if multiplied by a real constant, which depends on the overtone $n$. This clearly suggests a new form for $\Pi_{n}$.

\section{Conclusions}

Our analytical results provide that the dispersion relations for large frequencies are the same for all the three perturbation sectors and suggest that the QNM spectra for large $d$ are identical. Our numerical results confirm the eikonal limit for tensor-type gravitational perturbation. They are strong indicators that Eq. (4.3) can describe qualitatively but does not account for the correct quantitative behavior for vectorial-type and scalar-type gravitational perturbations.

\section{Acknowledgements}

JM thanks Fundação Universidade Federal do ABC (UFABC) for a grant. ASM and VTZ thank Conselho Nacional de Desenvolvimento Científico e Tecnológico (CNPq) - Brazil for grants. 

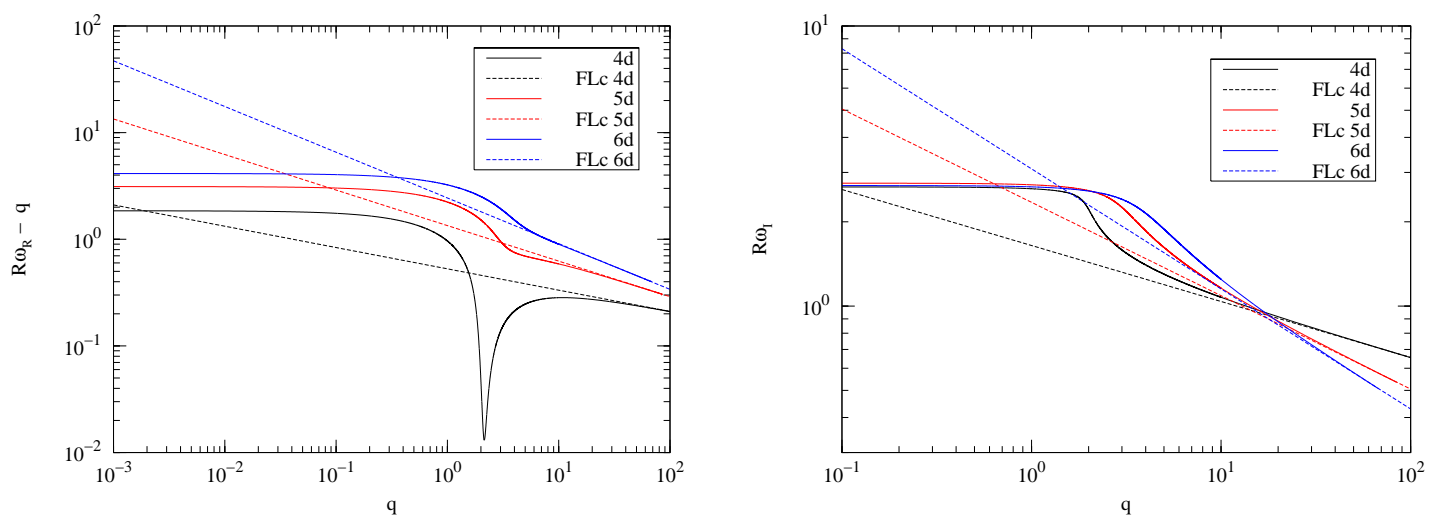

Figure 1: Numerical results for the fundamental vectorial QNM frequencies of a $r_{+} / R=1$ black hole. Left panel: real component $\omega_{R}$ (top to bottom are $d=6,5,4$ ). Right panel: imaginary component $\omega_{I}$ (top to bottom for $q>20$ corresponds to $d=4,5,6$ ). Dotted lines are the analytical prediction, corrected by a prefactor $a$.

\section{References}

[1] J. M. Maldacena, The large N limit of superconformal field theories and supergravity, Adv. Theor. Math. Phys. 2, 231 (1998).

[2] G. T. Horowitz and V. E. Hubeny, Quasinormal modes of AdS black holes and the approach to thermal equilibrium, Phys. Rev. D 62, 024027 (2000).

[3] P. K. Kovtun and A. O. Starinets, Quasinormal modes and holography, Phys. Rev. D 72, 086009 (2005) [arXiv:hep-th/0506184].

[4] A. S. Miranda, J. Morgan and V. T. Zanchin, Quasinormal modes of plane-symmetric black holes according to the AdS/CFT correspondence, JHEP 0811, 030 (2008) [arXiv:hep-th/0809.0297].

[5] J. P. S. Lemos, Two-dimensional black holes and planar general relativity, Class. Quant. Grav. 12, 1081 (1995) [arXiv:gr-qc/9407024].

[6] C. G. Huang and C. B. Liang, A torus-like black hole, Phys. Lett. A 201, 27 (1995).

[7] H. Kodama, A. Ishibashi and O. Seto, Brane world cosmology: gauge-invariant formalism for perturbation, Phys. Rev. D 62, 064022 (2000) [arXiv:hep-th/0004160].

[8] H. Kodama and A. Ishibashi, A master equation for gravitational perturbations of maximally symmetric black holes in higher dimensions, Prog. Theor. Phys. 110, 701 (2003) [arXiv:hep-th/0305147].

[9] J. Morgan, V. Cardoso, A. S. Miranda, C. Molina, and V. T. Zanchin, Gravitational quasinormal modes of AdS black branes in d spacetime dimensions [arXiv:hep-th/0907.5011].

[10] J. Morgan, V. Cardoso, A. S. Miranda, C. Molina, and V. T. Zanchin, Quasinormal modes of black holes in anti-de Sitter space: A numerical study of the eikonal limit, Phys. Rev. D 80, 024024 (2009) [arXiv:hep-th/0906.0064].

[11] G. T. Horowitz and V. E. Hubeny, Quasinormal modes of AdS black holes and the approach to thermal equilibrium, Phys. Rev. D 62, 024027 (2000) [arXiv:hep-th/9909056].

[12] G. Festuccia and H. Liu, A Bohr-Sommerfeld quantization formula for quasinormal frequencies of AdS black holes, [arXiv:gr-qc/0811.1033]. 